\title{
Avaliação e desenvolvimento da compreensão de leitura em universitários
}

\author{
Evaluation and development of college \\ students' comprehension
}

Adriana Rosecler ALCARÁ

Acácia Aparecida Angeli dos SANTOS²

\begin{abstract}
Resumo
Este estudo objetivou verificar os efeitos de um programa de remediação da compreensão de leitura. O delineamento usado foi quase-experimental, com pré-teste, intervenção e pós-teste. Participaram da pesquisa 41 universitários, com idade de 18 a 51 anos, sendo 22 do Grupo Experimental e 19 do Grupo Controle. Foi utilizado o teste de Cloze no prée no pós-teste. Os resultados indicaram que os estudantes mostraram desempenho superior no pós-teste, visto que houve um incremento na pontuação em mais da metade dos participantes, mas que tal melhora foi insuficiente para mudar o nível de compreensão, na maioria dos casos. Pode-se concluir que as atividades desenvolvidas foram relevantes, principalmente porque favoreceram a conscientização dos estudantes sobre a importância da compreensão de leitura e do estudo eficiente.
\end{abstract}

Palavras-chave: Compreensão de leitura; Programa de intervenção; Teste de Cloze.

\begin{abstract}
The purpose of this study was to evaluate the effects of a program for the remediation of reading comprehension. The experimental design was quasi-experimental, with pre-test and post-test interventions. Forty-one college students aged 18 to 51 years participated in the study, which were divided into two groups: 22 in the Experimental Group and 19 in the Control Group. We used a cloze test in the pre- and post-tests. The results indicated that the students showed improved performance in the post-test as more than half of the participants increased their scores, but this improvement was insufficient to change the level of reading comprehension in most cases. It may be concluded that the activities were relevant mainly because they favored awareness of students about the importance of reading comprehension and effective studying.
\end{abstract}

Keywords: Reading comprehension; Intervention program; Cloze testing.

$\boldsymbol{\nabla} \mathbf{v} \boldsymbol{v}$

1 Universidade Estadual de Londrina, Departamento de Ciências da Informação, Programa de Pós-Graduação em Ciência da Informação. Campus Universitário, Caixa Postal 6001, 86051-980, Londrina, PR, Brasil. Correspondência para/Correspondence to: A. R. ALCARÁ. E-mail: <alcara@uel.br>

2 Universidade São Francisco, Programa de Pós-Graduação em Psicologia. Itatiba, SP, Brasil.

Artigo baseado na tese de A. R. ALCARÁ, intitulado "Compreensão de leitura, estratégias de aprendizagem e motivação em universitários: estudo de validade de medidas". Universidade São Francisco, 2012. 
A leitura está presente em diferentes momentos da vida do ser humano e constitui ferramenta essencial para a construção social e cognitiva do indivíduo, contribuindo de forma efetiva para sua inserção na sociedade (Santos, Boruchovitch, \& Oliveira, 2009). A respeito da leitura, Santos e Oliveira (2004) observam que ela não é uma temática restrita a uma única área de estudo, e que há muitas abordagens ou correntes teóricas às quais se recorre para a compreensão do fenômeno. No entanto, grande parte dos modelos teóricos é convergente, ao considerarem a leitura como um recurso inquestionável para o saber e para o desenvolvimento do pensamento e, ainda, ao entenderem a compreensão como um processo que envolve a apreensão e a abstração do significado da leitura.

De acordo com Sternberg (2008), a leitura é um processo complexo, que envolve percepção, memória, inferência, dedução e processamento estratégico, sendo por isso considerada uma atividade cognitiva por excelência. Referindo-se à leitura, Leybaert, Alégria, Deltour e Skinkel (1997) também a concebem como uma atividade complexa, em que há a interação de diferentes mecanismos de comportamentos, a saber: identificação de letras; reconhecimento de palavras e seus significados; e integração sintática e semântica. Santos, Primi, Taxa e Vendramini (2002), fundamentados em Smith (1989), salientam que o ato de ler refere-se ao processo no qual a interpretação do que é lido depende não só do que está impresso, mas também das hipóteses do próprio leitor, que as formula com base no seu conhecimento prévio, e das conexões intertextuais estabelecidas, que permitirão a leitura significativa. Sendo assim, o ato de ler significa compreender o que foi lido.

Nessa perspectiva, ressalta-se que a compreensão de leitura requer vários processos cognitivos inter-relacionados, dentre os quais se destacam os processos básicos de leitura, como o reconhecimento e extração do significado das palavras impressas. Como ressaltam Kintsch e van Dijk (1978), esses requisitos não são suficientes, pois uma compreensão eficiente exige habilidades linguísticas gerais, memória, capacidade de realizar inferências 64 e conhecimento de mundo, fatores que juntos contribuem para a construção de uma representação macroestrutural do texto. Segundo os autores, a aprendizagem requer a absorção de conhecimentos que serão utilizados em outros contextos, sendo portanto necessária a sua retenção na memória. Por meio de pesquisas, os autores verificaram que leitores com alto nível de conhecimento anterior apresentavam melhor desempenho na compreensão de leitura, quando comparados aos leitores com baixo nível de conhecimento anterior.

Assim, segundo Vicentelli (2004), espera-se que o estudante universitário seja capaz de ler segundo um propósito, que crie estratégias próprias para compreensão de leitura, sintetize as informações a partir do texto e de sua própria experiência, elabore inferências e aplique o conhecimento adquirido para a resolução de problemas. A autora ainda ressalta que o sucesso em um curso superior está relacionado com a maturidade de leitura do estudante, o que envolve habilidades como compreensão, ritmo, concentração, flexibilidade, criticidade e criatividade.

No caso específico dos universitários, a leitura é indispensável, pois o acesso ao conteúdo das várias disciplinas e da produção científica acontece, principalmente, por meio dela. Ademais, enquanto leitor competente, o estudante compreende e se apropria de forma crítica das informações contidas nos textos, tanto no período de sua formação, como posteriormente em sua atuação profissional (Santos, 1997; Witter, 1997).

No entanto, em se tratando da compreensão de leitura, sabe-se que ela tem sido apontada como uma das principais dificuldades enfrentadas pelos estudantes em todas as etapas de escolaridade. No ensino superior, supõe-se que o estudante apresente um nível de leitura independente e que seja capaz de compreender e analisar de forma criativa as informações adquiridas (Cantalice \& Oliveira, 2009). Porém, as pesquisas realizadas com universitários têm evidenciado o baixo nível de compreensão de leitura e indicam que isso interfere diretamente no desempenho acadêmico (Oliveira, Santos, \& Primi, 2003; Oliveira \& Santos, 2006; Santos et al., 2002; Santos, Suehiro, \& Oliveira, 2004). 
Santos (2004) destaca algumas características dos leitores com dificuldades na compreensão de leitura, tais como: falhas no processo de decodificação, carências de vocabulário, leitura oral pobre, deficiência de integração das informações e de memória, falta de estratégias de aprendizagem adequadas e falta de motivação para a leitura. Segundo a autora, essas características reproduzem um círculo vicioso, em que os estudantes que apresentam dificuldades de compreensão evitam as atividades de leitura, não alcançando a prática necessária para uma leitura fluente, fazendo com que, consequentemente, o envolvimento e o nível de motivação para essas atividades tendam a diminuir.

Ainda, de acordo com Santos (1997), o leitor universitário, no decorrer de suas atividades acadêmicas, precisa assimilar uma gama variada de informações e isso pode promover nele a consciência da necessidade de melhorar suas habilidades para a compreensão de leitura. Assim, evidencia-se a importância de programas de diagnóstico e intervenção que visem identificar os níveis de compreensão e, posteriormente, melhorar as condições de leitura dos estudantes. Complementando, Solé (1998) salienta que devem ser ensinadas estratégias para a compreensão de leitura, possibilitando ao estudante a habilidade de ler um texto de maneira eficiente.

Dada a relevância da compreensão de leitura para o processo de aprendizagem, faz-se necessário abordar as possibilidades de como avaliá-la. Ao refletir sobre a avaliação da compreensão leitora, Oliveira, Boruchovitch e Santos (2009) enfatizam que essa não é uma tarefa fácil, uma vez que o produto que resulta dessa avaliação nem sempre pode ser observado de forma direta e objetiva. Segundo as autoras, a literatura registra a complexidade de se elaborarem instrumentos adequados para mensurar a compreensão leitora. A avaliação dessa habilidade requer instrumentos que forneçam resultados consistentes quanto à capacidade de compreensão e não apenas à produção textual. Geralmente os testes que visam avaliar essa habilidade não englobam os diversos aspectos envolvidos nesse processo, uma vez que focalizam apenas parte do comportamento relacionado à leitura e sua compreensão.

Uma das possibilidades adotadas para avaliar a compreensão de leitura é a técnica de Cloze, que tem sido objeto de atenção de estudiosos desde sua proposição por Taylor (1953) até os anos recentes, em que estudos mostram sua adequação como medida rápida e confiável da compreensão de leitura (Bellon-Harn, Credeur-Pampolina, \& LeBoeuf, 2013; Briere, Clausing, Senko, \& Purcell, 1978; Gellert \& Elbro, 2012; Kroeger, Burton, \& Preston 2009; Shahnazari-Dorcheh, Roshan, \& Hesabi, 2012). No Brasil, o Cloze tem sido bastante usado para o diagnóstico do nível de compreensão de leitura em universitários, visto que consiste na eliminação de alguns vocábulos do texto, que são substituídos por traços. Durante a aplicação do teste, o participante deve completar as lacunas com as palavras que julgar mais adequadas para completar o sentido do texto (Oliveira, et al., 2009; Oliveira \& Santos, 2008; Santos \& Oliveira, 2004).

Quanto às formas de correção dos textos preparados com a técnica de Cloze, tem-se a literal e a sinônima (Bitar, 1989). A correção literal considera como acerto o preenchimento correto da palavra exata que foi omitida, respeitando-se a grafia e a acentuação gráfica. Já a correção sinônima considera como correto o preenchimento da lacuna não apenas com a palavra que foi excluída, mas também com um sinônimo. Nesse caso, é preciso utilizar um critério, como por exemplo, um bom dicionário de língua portuguesa, para a seleção dos termos sinônimos. Ressalta-se que, no presente estudo, optou-se pela correção literal, visto que ela tem se mostrado apropriada para aferição da compreensão de leitura especialmente com universitários, pois minimiza o erro decorrente da subjetividade que pode ocorrer na interpretação da correção de palavras sinônimas (Oliveira et al., 2003; Sampaio \& Santos, 2002).

Para a interpretação dos escores obtidos com o teste de Cloze, pode-se ou utilizar a média e o desvio-padrão da pontuação obtida pelos participantes, ou utilizar os níveis estabelecidos por Bormuth (1968). O primeiro nível, denominado nível de frustração, exige o acerto de $44,0 \%$ das lacunas 
preenchidas, o que classifica o participante como leitor que não compreende a informação lida. No segundo nível, denominado instrucional, com acerto entre 44,1 e $57,0 \%$, considera-se que o leitor demonstra uma abstração apenas suficiente para a compreensão e necessita da mediação do professor. Por fim, o terceiro nível, conhecido como independente, exige pontuação superior a 57,0\% de acertos, avaliando-se que tais leitores possuem uma compreensão crítica, criativa e autônoma (Oliveira et al., 2009; Oliveira \& Santos, 2008).

De acordo com Santos (2004), a técnica de Cloze tem se destacado por sua utilidade tanto para o diagnóstico como para o desenvolvimento da compreensão de leitura, além de ser uma técnica que une aspectos de praticidade, economia de tempo e recursos. Nessa perspectiva, embora ainda em número reduzido, algumas pesquisas brasileiras com estudantes universitários foram desenvolvidas com o objetivo de avaliar a eficácia de programas diagnósticos e interventivos em leitura (Alves, 2010; Hussein, 2008; Sampaio \& Santos, 2002; Santos, 1997). Os resultados evidenciaram melhora na compreensão de leitura, no desempenho acadêmico e nos hábitos de estudo dos participantes. Além disso, após a intervenção, os participantes mostraram-se mais conscientes quanto à importância da leitura e das próprias dificuldades, bem como mais conhecimento em relação às estratégias necessárias para uma leitura eficiente.

Ainda, em vários outros estudos com universitários, vêm sendo utilizados textos preparados com a técnica de Cloze para a avaliação da compreensão de leitura. Podem-se citar, por exemplo, Boruchovitch, Santos e Oliveira (2007), Calderón Ibañez e Quijano Peñuela (2010), Cantalice e Oliveira (2009), Difabio de Anglat (2008), Martins, Santos e Bariani (2005), Oliveira (2011) e Silva e Santos (2004), entre outros. Em síntese, os resultados dessas pesquisas constataram que o nível de compreensão de leitura em estudantes universitários ingressantes está aquém do desejado, e que a compreensão de leitura relaciona-se com o desempenho acadêmico.

Assim, a técnica de Cloze tem se mostrado adequada tanto como medida de compreensão de 66 leitura, quanto como recurso para auxiliar no desen- volvimento das habilidades linguísticas dos estudantes em programas específicos de intervenção ou, ainda, como recurso pedagógico em disciplinas diversas. Considerando-se os aspectos aqui apresentados, especialmente no que se refere à importância da leitura competente em estudantes do ensino superior, o presente estudo propõe-se a verificar os efeitos da aplicação de um programa de intervenção para o desenvolvimento da compreensão de leitura em universitários.

\section{Método}

Esta pesquisa foi desenvolvida com delineamento quase-experimental, com pré e pós-teste, utilizando-se de um programa de intervenção. Seu objetivo foi aprimorar o desempenho dos participantes no que se refere às habilidades para a compreensão de leitura.

\section{Participantes}

Os participantes desta pesquisa foram divididos em Grupo Experimental (GE) e Grupo Controle (GC).

O Grupo Experimental foi composto por estudantes da $1^{\text {a }}$ série do curso de Biblioteconomia, visto que sua coordenação disponibilizou um espaço curricular para que o programa de intervenção fosse desenvolvido. Dos 49 estudantes matriculados nessa série, 46 participaram do GE. Destes, foram excluídos 24, por não atenderem aos critérios de participação pré-estabelecidos: (a) participação no pré e pós-teste; (b) participação em pelo menos quatro das cinco sessões da intervenção. Assim, a amostra final do GE foi constituída por 22 estudantes, sendo $16(72,7 \%)$ do sexo feminino e 6 $(27,3 \%)$ do sexo masculino. A média de idade foi de 25 anos e 2 meses (Desvio-Padrão - DP = 8,47), sendo a mínima 18 e a máxima 51 anos.

Para a composição do Grupo Controle foram selecionados os estudantes da $1^{\text {a }}$ série do curso Arquivologia, com perfil equivalente aos estudantes do curso de Biblioteconomia, tanto em termos de interesses como de competências quando do ingres- 
so na universidade. Assim, o GC ficou constituído por 19 estudantes, sendo 11 (57,9\%) do sexo feminino e $8(42,1 \%)$ do sexo masculino. A média de idade da amostra do GC foi de 27 anos e 7 meses $(D P=9,07)$, variando de 18 a 45 anos.

\section{Instrumentos}

Para a avaliação da compreensão de leitura, tanto no pré como no pós-teste, foi utilizado o teste de Cloze, com o texto "Desentendimento" de Luis Fernando Veríssimo (1995). O texto, que contém originalmente 250 vocábulos, foi reestruturado com a omissão de todo quinto vocábulo, num total de 46 lacunas a serem preenchidas. A pontuação variou de 0 a 46, sendo atribuído 1 ponto para cada acerto e 0 para os erros. O critério para a pontuação foi o literal, que considera como correta a palavra exata que foi omitida.

As propriedades psicométricas do teste foram identificadas por Santos et al. (2002), sendo que a precisão do instrumento, medida pelo alfa de Cronbach, apresentou um coeficiente de 0,82. Os autores também observaram um bom ajuste ao modelo de dois parâmetros da Teoria de Resposta ao Item - discriminação e dificuldade -, sendo a média de ID de 0,487 . Outros estudos realizados com esse mesmo teste confirmaram evidências de validade e precisão como medida de avaliação da compreensão de leitura (Oliveira \& Santos, 2008).

Ainda, no decorrer do programa de intervenção, foram utilizados diferentes materiais preparados para as atividades reflexivas e práticas da intervenção. Primeiramente foram preparados slides com uma abordagem dos aspectos relativos às habilidades cognitivas e metacognitivas necessárias à compreensão de leitura. Em seguida, para as atividades práticas, foram selecionados três textos disponíveis na literatura e preparados com a técnica de Cloze, em diferentes variações.

\section{Procedimentos}

Após a aprovação do Comitê de Ética em Pesquisa da Universidade São Francisco (Protocolo $\mathrm{n}^{\circ}$ 0330.0.000.142-10), foram agendadas as aplicações do pré-teste, as sessões de intervenção e o pós-teste, mediante a concordância do Colegiado e professores dos cursos. As aplicações do pré- e pós-teste bem como as atividades interventivas ocorreram de forma coletiva, em período normal de aula.

As sessões do programa de intervenção realizado com o Grupo Experimental foram desenvolvidas no horário de uma das disciplinas do curso, durante o mês de agosto de 2011. Foram realizadas cinco sessões, as quatro primeiras com duração de duas horas/aula, e a quinta sessão com duração de quatro horas/aula, perfazendo um total de 12 horas/aula destinadas ao programa de intervenção.

De forma geral, as sessões da intervenção foram divididas em três partes. Uma parte era destinada à apresentação e reflexão em torno dos conteúdos relacionados à compreensão de leitura e às estratégias de aprendizagem, tais como: a importância da leitura e sua compreensão para as atividades acadêmicas, formação e atuação profissional futura; os processos metacognitivos necessários à compreensão de leitura; as principais dificuldades para a compreensão; os tipos de leitura; a técnica de Cloze e sua utilização para o diagnóstico e desenvolvimento da compreensão de leitura, entre outros. Na outra parte da sessão, eram propostas atividades práticas com o uso de textos preparados com a técnica de Cloze. Vale mencionar que, antes do início de cada atividade eram dadas as orientações necessárias para a execução da tarefa. Por fim, a sessão era encerrada com a correção do exercício prático e uma discussão a respeito dos procedimentos adotados, das dificuldades encontradas e da percepção dos estudantes sobre as tarefas desenvolvidas. Ao final do programa, foi realizada uma avaliação das atividades desenvolvidas no decorrer de todas as sessões.

Para o planejamento e desenvolvimento das atividades também se considerou uma sequência progressiva do nível de dificuldade do Cloze. Dessa forma, primeiramente utilizou-se um texto de Cloze gradual, com três alternativas de respostas. Já o segundo o texto foi elaborado com a técnica de Cloze limitado, em que todas as palavras omitidas estavam dispostas aleatoriamente, em uma folha 
anexada ao texto. Na sequência, o terceiro texto foi preparado com a técnica de Cloze com a omissão de todo décimo vocábulo. Na última sessão, aplicou-se o teste de Cloze ao texto "Desentendimento" (pós-teste), com as omissões a cada quinto vocábulo.

Foram utilizados testes da estatística não paramétrica especialmente em razão do tamanho dos dois grupos, GE e GC. Assim, para a comparação do desempenho dos participantes dos dois grupos, recorreu-se ao teste de $U$ de Mann-Whitney. Para a análise do desempenho dos participantes no pré e pós-testes utilizou-se a prova de Wilcoxon Signed Ranks (Teste de Sinal Z).

\section{Resultados}

Inicialmente, foi realizada a comparação do desempenho dos participantes dos dois grupos no momento do pré-teste, por meio do teste de $\mathrm{U}$ de Mann-Whitney. Os dados mostraram a inexistência de diferenças significativas entre os eles no início do estudo ( $U=199,5 ; p=0,803)$. Na sequência, buscou-se identificar se havia diferenças intragrupos, recorrendo-se à prova de Wilcoxon Signed Ranks (Teste de Sinal Z), pela qual foram identificados os participantes que melhoraram o desempenho, os que permaneceram na mesma posição e os que pioraram. A Tabela 1 apresenta os resultados obtidos para o GE e GC:

Os dados da Tabela 1 revelam melhora significativa no desempenho dos estudantes em ambos os grupos. As médias por rank mostram que 12 estudantes melhoraram seu desempenho do pré para o pós-teste no GE, enquanto que no GC essa melhora ocorreu para 15 estudantes.
Além da comparação intragrupo, optou-se também por fazer uma análise do nível de compreensão de leitura, a partir do número de acertos obtidos pelos estudantes. Para essa análise, utilizou-se a proposta de níveis de compreensão de leitura elaborada por Bormuth (1968). De acordo com esse autor, conforme já mencionado, o total de acertos obtido pelos estudantes pode ser classificado da seguinte forma: nível de frustração (até $44 \%$ de acertos); nível instrucional (de 44 a 57\% de acertos) e nível independente (acima de $57 \%$ de acertos). Ressalta-se que no Teste de Cloze utilizado nesta pesquisa a pontuação variou de 0 a 46 acertos. A Tabela 2 mostra o total de acertos obtidos por cada um dos participantes do GE e GC e o nível de compreensão de leitura no pré- e pós-teste, a partir número de acertos obtidos.

A partir dos dados da Tabela 2, pode-se observar que, de forma geral, a grande maioria dos estudantes encontrava-se no nível instrucional nos dois momentos da pesquisa. Embora 12 estudantes do GE tenham apresentado melhora na pontuação do pós-teste, conforme já visto anteriormente na Tabela 1, apenas seis aumentaram suficientemente para mudar de nível, considerando-se a proposta de Bormuth (1968). Separando os estudantes por níveis, têm-se os seguintes resultados: Dos cinco estudantes que se encontravam no nível de frustração no pré-teste, três mudaram de nível no pósteste, sendo que dois passaram para o nível instrucional, e um passou para o nível independente. Dos 16 estudantes com nível instrucional no pré-teste, apenas três passaram para o nível independente no pós-teste, enquanto os demais permaneceram no mesmo nível. Quanto ao nível independente no pré-teste, apenas um estudante obteve pontuação para tal e permaneceu nesse mesmo nível no pós-teste.

Tabela 1

Comparação intragrupo do desempenho dos participantes do Grupo Experimental e Grupo Controle no pré- e pós-testes

\begin{tabular}{|c|c|c|c|c|c|c|c|c|c|}
\hline \multirow{2}{*}{ Medida } & \multirow{2}{*}{ Ranks } & \multicolumn{4}{|c|}{ Grupo Experimental } & \multicolumn{4}{|c|}{ Grupo Controle } \\
\hline & & $\mathrm{N}$ & M por Ranks & $\mathrm{Z}$ & $p$ & $\mathrm{~N}$ & M por Ranks & $\bar{Z}$ & $p$ \\
\hline Cloze (pós-teste) & Negativos & 7 & 6,64 & & & 2 & 2,00 & & \\
\hline \multirow[t]{2}{*}{ Cloze (pré-teste) } & Positivos & 12 & 11,96 & $-1,961$ & 0,050 & 15 & 9,93 & $-3,454$ & 0,001 \\
\hline & Empates & 3 & & & & 2 & & & \\
\hline
\end{tabular}


Tabela 2

Total de acertos e nível de compreensão de leitura dos participantes de ambos os grupos

\begin{tabular}{|c|c|c|c|c|c|c|c|c|}
\hline \multirow{3}{*}{ Participantes } & \multicolumn{4}{|c|}{ Grupo Experimental } & \multicolumn{4}{|c|}{ Grupo Controle } \\
\hline & \multicolumn{2}{|c|}{ Total de acertos } & \multicolumn{2}{|c|}{ Nível de comprensão } & \multicolumn{2}{|c|}{ Total de acertos } & \multicolumn{2}{|c|}{ Nível de comprensão } \\
\hline & Pré & Pós & Pré & Pós & Pré & Pós & Pré & Pós \\
\hline 1 & 20 & 23 & FRS & INS & 16 & 20 & FRS & FRS \\
\hline 2 & 26 & 23 & INS & INS & 25 & 27 & INS & IND \\
\hline 3 & 24 & 32 & INS & IND & 24 & 23 & INS & INS \\
\hline 4 & 22 & 22 & INS & INS & 26 & 26 & INS & INS \\
\hline 5 & 25 & 28 & INS & IND & 21 & 26 & INS & INS \\
\hline 6 & 21 & 26 & INS & INS & 17 & 21 & FRS & INS \\
\hline 7 & 24 & 23 & INS & INS & 26 & 29 & INS & IND \\
\hline 8 & 21 & 21 & INS & INS & 20 & 20 & FRS & FRS \\
\hline 9 & 21 & 30 & INS & IND & 22 & 24 & INS & INS \\
\hline 10 & 19 & 27 & FRS & IND & 24 & 26 & INS & INS \\
\hline 11 & 18 & 23 & FRS & INS & 29 & 32 & IND & IND \\
\hline 12 & 21 & 22 & INS & INS & 24 & 29 & INS & IND \\
\hline 13 & 22 & 22 & INS & INS & 17 & 19 & FRS & FRS \\
\hline 14 & 24 & 23 & INS & INS & 14 & 22 & FRS & INS \\
\hline 15 & 23 & 25 & INS & INS & 21 & 23 & INS & INS \\
\hline 16 & 26 & 22 & INS & INS & 25 & 26 & INS & INS \\
\hline 17 & 25 & 24 & INS & INS & 27 & 26 & IND & INS \\
\hline 18 & 16 & 14 & FRS & FRS & 17 & 21 & FRS & INS \\
\hline 19 & 27 & 29 & IND & IND & 13 & 15 & FRS & FRS \\
\hline 20 & 20 & 19 & FRS & FRS & - & - & - & - \\
\hline 21 & 21 & 25 & INS & INS & - & - & - & - \\
\hline 22 & 23 & 24 & INS & INS & - & - & - & - \\
\hline
\end{tabular}

Nota: FRS: Frustração; INS: Instrucional; IND: Independente.

Quanto aos participantes do GC, os dados da Tabela 2 mostram que um pouco mais da metade dos estudantes encontrava-se no nível instrucional. Dos 15 quinze estudantes que apresentaram melhora na pontuação do pós-teste, apenas seis aumentaram no nível de compreensão. Dos sete estudantes que estavam no nível de frustração no pré-teste, três passaram para o nível instrucional no pós-teste e quatro permaneceram no nível de frustração. Dos 10 estudantes com nível instrucional no pré-teste, três passaram para o nível independente no pós-teste. Dos dois estudantes que estavam no nível independente no pré-teste, um diminuiu para o nível instrucional, e o outro permaneceu no mesmo nível no pós-teste.

\section{Discussão}

Considerando-se o propósito do estudo de avaliar os efeitos do programa de intervenção na compreensão de leitura dos estudantes, o fato de ter identificado que os grupos (experimental e de controle) eram comparáveis foi essencial para as análises realizadas. Assim, os resultados do teste $U$ de Mann-Whitney foram indicativos de que o ponto de partida de ambos os grupos foi igual no que se refere ao nível das habilidades relativas à compreensão de leitura.

No tocante à comparação intragrupo dos estudantes no pós-teste do Cloze, os resultados mostraram melhoras significativas dos estudantes nos dois grupos. No que se refere ao GE, pode-se supor que as atividades interventivas estimularam uma reflexão em torno da relevância e da contribuição da leitura, com compreensão para o progresso acadêmico e atuação futura dos estudantes. Além disso, percebe-se que as atividades interventivas com os textos preparados com a técnica de Cloze podem ter aprimorado as habilidades dos estudantes, levando-se em conta que mais da metade deles apresentou melhora de desempenho no pós-teste. Esses resultados são similares aos obtidos 
em outros estudos, que também empregaram o Cloze como técnica em programas de intervenção para aprimoramento da leitura e escrita (Hussein, 2008; Sampaio \& Santos, 2002; Santos, 1997).

Quando analisados os níveis de compreensão a partir da pontuação obtida no Teste de Cloze antes e depois do programa de intervenção, também se percebeu um incremento na pontuação em mais da metade dos participantes do GE. No entanto, apenas seis dos estudantes tiveram melhora suficiente a ponto de mudar de nível de compreensão. Vale ainda mencionar que cinco estudantes estavam no nível de frustração (não compreende a informação lida) no pré-teste, sendo que três deles melhoraram a pontuação e apresentaram uma ascensão no nível de compreensão. Todavia, no GC também houve melhora no desempenho dos estudantes no momento do pós-teste, sendo que seis apresentaram ascensão no nível de compreensão.

A partir desses dados, não se pode conferir a melhora dos resultados no pós-teste apenas ao efeito das atividades realizadas durante o programa de intervenção, uma vez que o GC também mostrou evolução no desempenho em compreensão de leitura. Uma possível explicação para o fato do GC também ter apresentado resultados superiores no pós-teste pode ser atribuída ao próprio Cloze, que se mostra útil tanto para o diagnóstico, como para o desenvolvimento da compreensão de leitura, conforme destaca Santos (2004). Além disso, o contato e o preenchimento do instrumento em dois momentos (pré e pós-teste) podem ter propiciado maior segurança aos participantes no momento de responder ao teste, levando-se em conta que no pós-teste eles já conheciam o formato da medida.

A inexistência de efeitos mais significativos na compreensão de leitura dos participantes do GE após as atividades de intervenção também fez parte dos resultados de estudos interventivos anteriormente realizados com universitários. Podem-se citar, por exemplo, as pesquisas de Sampaio e Santos (2002) e Santos (1997). Contudo, mesmo tendo o respaldo desses estudos anteriores, levantam-se algumas possíveis causas para a falta de melhora de forma significativa na compreensão de leitura 70 na presente pesquisa. Primeiramente, destaca-se o fator tempo. Neste estudo, com exceção do último encontro, as demais sessões foram de apenas duas horas/aula, quando talvez fosse ideal fazer encontros semanais de quatro horas/aulas. Encontros mais demorados poderiam possibilitar que se desenvolvesse de forma mais detalhada cada uma das atividades práticas, assim como poderiam ensejar a reflexão em torno do processo adotado pelos estudantes ao realizarem aquelas atividades. Sobretudo, acredita-se também ser necessário um número maior de sessões. Considerando a complexidade dos processos envolvidos na compreensão de leitura, conclui-se que cinco sessões, como realizado neste estudo, não são suficientes para estimular e desenvolver as habilidades requeridas para uma compreensão adequada.

Conforme já mencionado no decorrer deste trabalho, a compreensão de leitura é um processo complexo, que demanda uma série de habilidades cognitivas que são inter-relacionadas, como por exemplo: reconhecimento de palavras e extração de significados; habilidades de memória; conhecimento prévio e de mundo; capacidade para realização de inferências; além do interesse e uso adequado de estratégias de leitura (Kintsch \& van Dijk, 1978; Santos \& Oliveira, 2004; Sternberg, 2008). Contudo, os resultados aqui trazidos mostram que o uso da técnica de Cloze pode ser uma alternativa que possibilita o manejo de muitos desses aspectos, de forma a favorecer o desenvolvimento da compreensão leitora.

Assim, para desenvolver a compreensão de leitura por meio do Cloze, os textos devem ser preparados a partir das diversas possibilidades de emprego da técnica. Segundo Oliveira et al. (2009), existem muitas variações da técnica que podem ser utilizadas para estruturar um texto. Dentre elas, as autoras mencionam os vários tipos de Cloze, a saber: lexical, gramatical, cumulativo, de múltipla escolha, labirinto, pareado, restringido, pós-leitura oral, interativo, entre outros. Vale resgatar que, na pesquisa aqui desenvolvida utilizou-se o Cloze gradual nos momentos do pré e pós-teste, e que, durante o programa de intervenção, foram empregadas outras três variações da técnica (Cloze múltipla escolha com três alternativas de respostas, 
Cloze limitado e Cloze com a omissão de todo décimo vocábulo).

Embora tenham sido utilizadas essas diferentes variações para as atividades de compreensão, isso não foi suficiente para mudar as habilidades dos estudantes. Percebeu-se que, se houvesse mais tempo destinado à realização dos exercícios com o Cloze, a sensibilização inicial promovida pelas atividades poderia resultar em ganhos mais expressivos no desempenho do pós-teste. Fundamenta-se esse aspecto no fato de que foi possível observar, no momento da apresentação das atividades em cada uma das sessões, interesse e entusiasmo por parte dos estudantes para entender o funcionamento da técnica e refletir sobre a importância da leitura para suas atividades acadêmicas. Em longo prazo, provavelmente, esse envolvimento tenderia a ter reflexos positivos no processo da aprendizagem esperada para o programa de intervenção.

Em relação ao tempo determinado para o programa de intervenção, cabem algumas considerações. Primeiramente, é necessário levar em conta o próprio contexto do curso em que foi realizada a intervenção: trata-se de curso noturno, composto majoritariamente por estudantes que trabalham ou estagiam durante o dia, que têm apenas a noite para se dedicar aos estudos. Dessa forma, eles não dispõem de tempo para participar de atividades fora do horário normal de aulas, de modo que o programa foi oferecido no período das aulas, utilizando-se da carga horária de disciplina da grade curricular, o que justifica a definição de apenas 12 horas para sua realização.

Retomando a discussão a respeito dos efeitos do programa de intervenção na compreensão de leitura dos estudantes, aventa-se a possibilidade de que pode ter havido, no mínimo, uma melhora no que diz respeito à conscientização sobre a importância do estudo e da leitura, bem como uma percepção quanto ao conhecimento das próprias dificuldades. A inferência sobre uma melhora qualitativamente perceptível é sustentada também com base na avaliação realizada junto aos estudantes, ao final do programa de intervenção. Tanto em conversas informais dos estudantes com a proponente desta pesquisa, como em um relato escrito realizado pelos participantes ao final do programa de intervenção, percebe-se uma melhora qualitativa e uma sensibilização maior para as questões relacionadas à compreensão de leitura. Vale lembrar que essa constatação caminha na mesma direção das pesquisas desenvolvidas por Sampaio e Santos (2002) e Santos (1997), que igualmente verificaram - após programas de intervenção com o uso da técnica de Cloze -, desempenhos qualitativamente superiores no que se refere aos hábitos de estudo e ao uso de estratégias de leitura.

Contudo, há que se considerar que os estudantes que participaram deste estudo, tanto no GE quanto no GC, de forma geral, ainda apresentam resultados insatisfatórios no que diz respeito à compreensão de leitura. Quando analisados os níveis de compreensão de acordo com a proposta de Bormuth (1968), percebe-se que em ambos os grupos o nível de compreensão está abaixo do esperado para o ensino superior. Esse resultado é recorrente em pesquisas realizadas com estudantes universitários, tais como as de Boruchovitch et al. (2007), Calderón Ibañez e Quijano Peñuela (2010), Martins et al. (2005), Oliveira (2011) e Silva e Santos (2004), entre outras. Aliado a isso, pode-se visualizar, a partir da análise do total de acertos e níveis de compreensão, que é mínima a frequência no nível independente, no qual se situam aqueles leitores que possuem uma compreensão crítica e autônoma do que leem. Esses dados mostram a falta de habilidades desejáveis para que um estudante universitário se torne um leitor com nível independente e, assim, realçam a importância de programas que visem remediar o problema.

Considerando-se o objetivo do presente trabalho, que consistiu em verificar os efeitos da aplicação de um programa de intervenção para a remediação da compreensão de leitura, enfatiza-se que, embora os resultados não tenham evidenciado impacto positivo na mudança de nível de compreensão leitora, as atividades desenvolvidas mostraram ser relevantes, principalmente no que diz respeito à conscientização dos estudantes quanto à importância das estratégias de aprendizagem para a compreensão de leitura e o estudo eficiente. Da mesma forma, destaca-se a contribuição das ativi- 
dades interventivas em promover nos estudantes a consciência em relação a suas dificuldades de leitura e aprendizagem.

Nesse sentido, confirma-se, mais uma vez, a relevância de programas que visem remediar os problemas relacionados às habilidades necessárias para a compreensão de leitura e o estudo competente. Reforça-se, assim, a necessidade de maior investimento nesses programas para os estudantes ingressantes na universidade, seja por parte dos professores e coordenadores de cursos, seja por iniciativa institucional. Destaca-se principalmente que o domínio dessas habilidades reflete-se não apenas no progresso e melhor desempenho na aprendizagem, mas também na atuação cidadã e profissional futura desses estudantes.

Assim, outros estudos com foco em programas interventivos são necessários para investigar de forma mais detalhada as atividades que possam contribuir para o ensino e a promoção de habilidades cognitivas e metacognitivas promotoras de uma leitura eficiente. Salienta-se a importância do oferecimento de programas de instrução no âmbito de projetos de ensino, pesquisa ou extensão, com vistas à obtenção de resultados em médio e longo prazo.

Ainda, não obstante as limitações já mencionadas, destacam-se novamente as dificuldades de ordem temporal, tendo em vista o contexto do próprio curso em que a intervenção foi realizada. Vale também salientar a questão de que estudantes de diferentes cursos foram comparados, o que aumenta a probabilidade de que vieses possam ter ocorrido, de forma a interferir no desempenho no pós-teste. Espera-se que novas investigações possam superar essas limitações.

Acrescentam-se também as questões relativas à dificuldade de mudança no desempenho dos estudantes, primeiramente por se tratar de um processo complexo, que envolve aspectos cognitivos, metacognitivos e afetivos. Aliado a isso, deve-se considerar que esse processo torna-se ainda mais complexo em razão das deficiências por eles apresentadas quando do ingresso na universidade. Dentre elas, podem-se mencionar a falta de conhe72 cimento quanto às estratégias de aprendizagem (que deveriam já ter sido ensinadas nas etapas anteriores da escolarização) e a falta de interesse e autocontrole sobre as questões relacionadas à aprendizagem, além das condições gerais em que o ensino fundamental e médio foram oferecidos.

\section{Referências}

Alves, S. M. L. (2010). Estratégias de compreensão leitora e de produção de resumo do gênero científico: aspectos textuais e cognitivos (Tese de doutorado não-publicada). Pontifícia Universidade Católica do Rio Grande do Sul, Porto Alegre.

Bellon-Harn, M. L., Credeur-Pampolina, M. E., \& LeBoeuf, L. (2013). Scaffolded-language intervention: Speech production outcomes. Communication Disorders Quarterly, 34(2), 120-132.

Bitar, M. L. (1989). Eficiência dos instrumentos de avaliação em leitura (Dissertação de mestrado não-publicada). Pontifícia Universidade Católica de São Paulo.

Bormuth, J. R. (1968). Cloze test readability: Criterion reference scores. Journal of Educational Measurement, 5, 189-196.

Boruchovitch, E., Santos, A. A. A., \& Oliveira, K. L. (2007). Análise da fidedignidade entre dois tipos de pontuação do teste de Cloze. Psicologia em Pesquisa, 1(1), 41-51.

Briere, E., Clausing, G, Senko, D., \& Purcell, E. (1978). A look at cloze testing across languages and levels. The Modern Language, 62(1/2), 23-26.

Calderón Ibañez, A., \& Quijano Peñuela, J. (2010). Características de comprensión lectora em estudiantes universitários. Jurídicas, 7(2), 123-151.

Cantalice, L. M., \& Oliveira, K. L. (2009). Estratégias de leitura e compreensão textual em universitários. Psicologia Escolar e Educacional, 13(2), 227-234.

Difabio de Anglat, H. (2008). El test cloze em la evaluacion de La comprension del texto informativo de nível universitario. Revista de Linguistica Teórica y Aplicada, 46(1), 121-137.

Gellert, A. S., \& Elbro, C. (2012). Cloze tests may be quick, but are they dirty? Development and preliminary validation of a Cloze test of a reading comprehension. Journal of Psychoeducational Assessment, 20(10), 1-13.

Hussein, C. L. (2008). Avaliação de treino de leitura compreensiva e crítica: estudo com universitários. Psicologia Escolar e Educacional, 12(2), 401-411.

Kintsch, W., \& Van Dijk, T. A. (1978). Toward a model of text comprehension and production. Psychological Review, 85(5), 363-394.

Kroeger, S. D., Burton, C., \& Preston, C. (2009). Integrating evidence-based practices in middle science reading. Teaching Exceptional Children, 41(3), 6-15. 
Leybaert, J., Alégria, J., Deltour, J. J., \& Skinkel, R. (1997). Aprender a ler: o papel da linguagem, da consciência fonológica e da escola. In J. Gregoire, \& B. Piérart. Avaliação problemas de leitura: os novos modelos teóricos e suas implicações diagnósticas (pp.143-166). Porto Alegre: Artes Médicas.

Martins, R. M. M, Santos, A. A. A., \& Bariani, I. C. D. (2005). Estilos cognitivos e compreensão leitora em universitários. Paidéia, 15(30), 57-68.

Oliveira, K. L. (2011). Considerações acerca da compreensão em leitura no ensino superior. Psicologia: Ciência e Profissão, 31(4), 690-701.

Oliveira, K. L., Boruchovitch, E., \& Santos, A. A. A. (2009). A técnica de Cloze na avaliação da compreensão em leitura. In A. A. A. Santos, E. Boruchovicht, \& K. L Oliveira (Orgs.), Cloze: um instrumento de diagnóstico e intervenção (pp.47-79). São Paulo: Casa do Psicólogo.

Oliveira, K. L., \& Santos, A. A. A. (2006). Compreensão de textos e desempenho acadêmico. Psic: Revista de Psicologia da Vetor Editora, 9(1), 19-27.

Oliveira, K. L., \& Santos, A. A. A. (2008). Estudo de intervenção para a compreensão em leitura na universidade. Interação em Psicologia, 12(2), 169-177.

Oliveira, K. L., Santos, A. A. A., \& Primi, R. (2003). Estudo das relações entre compreensão em leitura e desempenho em disciplinas na universidade. Interação em Psicologia, 7(1), 19-25.

Sampaio, I. S., \& Santos, A. A. A. (2002). Leitura e redação entre universitários: Avaliação de um programa de intervenção. Psicologia em Estudo, 9(1), 31-38.

Santos, A. A. A. (1997). Psicopedagogia no $3^{\circ}$ grau: avaliação de um programa de remediação em leitura e estudo. Pro-Posições, 8(1), 27-37.

Santos, A. A. A. (2004). O Cloze como técnica de diagnóstico e remediação da compreensão em leitura. Interação em Psicologia, 8(2), 217-226.

Santos, A. A. A., Boruchovitch, E., \& Oliveira, K. L. (2009). Cloze: um instrumento de diagnóstico e intervenção. São Paulo: Casa do Psicólogo.

Santos, A. A. A., \& Oliveira, K. L. (2004). A importância da compreensão em leitura para a aprendizagem de universitários. In E. Boruchovitch, \& J. A. Bzuneck (Orgs.), Aprendizagem: processos psicológicos e o contexto social na escola (pp.119-148). Petrópolis: Vozes.

Santos, A. A. A., Primi, R., Taxa, F., \& Vendramini, C. M. M. (2002). O teste de Cloze na avaliação da compreensão em leitura. Psicologia: Reflexão e Crítica, 15(3), 549-560.

Santos, A. A. A., Suehiro, A. C. B., \& Oliveira, K. L. (2004). Habilidades em compreensão da leitura: um estudo com estudantes de psicologia. Estudos de Psicologia, 21(2), 29-41.

Shahnazari-Dorcheh, M., Roshan, S., \& Hesabi, A. (2012). What is the optimum length of a cloze test? International Journal of English Linguistics, 2(5), 2012, 142-153.

Silva, M. J. M., \& Santos, A. A. A. (2004). A avaliação da compreensão em leitura e o desempenho acadêmico de universitários. Psicologia em Estudos, 9(3), 459-467.

Smith, F. (1989). Compreendendo a leitura: uma análise psicolinguística da leitura e do aprender a ler ( $4^{\mathrm{a}} \mathrm{ed}$.). Porto Alegre: Artes Médicas.

Solé, I. (1998). Estratégias de aprendizagem (6 $6^{\mathrm{a}}$ ed.). Porto Alegre: Artmed.

Sternberg, R. J. (2008). Psicologia cognitiva (4 ${ }^{\mathrm{a}}$ ed.). Porto Alegre: Artmed.

Taylor, W. L. (1953). Cloze procedure: A new tool for measuring read-ability. Journalism Quartely, 30, 415-433.

Veríssino, L. F. (1995). Desentendimento. ĺcaro: Revista de Bordo da Varig, 12(136), 11.

Vicentelli, H. (2004). La universidad como agente promotor de la lectura, en el contexto de la sociedad venezolana. Psicologia Escolar e Educacional, 8(1), 29-33.

Witter, G. P. (1997). Psicologia, leitura e universidade. Campinas: Alínea.

Recebido: outubro 26, 2012

Versão final: novembro 21, 2013

Aprovado: dezembro 4, 2014 
